\title{
Infiltrative (sinusoidal) and hepatitic patterns of injury in acute cellular rejection in liver allograft with clinical implications
}

\author{
Iram Siddiqui ${ }^{1}$, Nazia Selzner ${ }^{2}$, Sara Hafezi-Bakhtiari ${ }^{1}$, Max A Marquez ${ }^{2}$ and \\ Oyedele A Adeyi ${ }^{1,2}$ \\ ${ }^{1}$ Laboratory Medicine, University of Toronto, Toronto, ON, Canada and ${ }^{2}$ Multiorgan Transplant Programs, \\ University Health Network, University of Toronto, Toronto, ON, Canada
}

\begin{abstract}
Acute cellular rejection post liver transplant occurs most commonly but not exclusively in the first year. In this study, we report two patterns: sinusoidal infiltrative and hepatitic, which are not considered in the Banff system. We describe their presentation, response to Solu-Medrol, and compare these to the typical moderate-severe acute cellular rejection. Patients transplanted from 2007 to 2012 at University Health Network, who had biopsyproven rejection in the first year, were studied. Baseline transaminases and bilirubin, time of acute cellular rejection, follow-up, and treatment responses were analyzed. A total of 407 biopsies were received, of which 77 had diagnosis of acute cellular rejection with rejection activity index 5 or above; 49 from viral hepatitis patients were excluded. Twenty-eight were included; 15/28 (54\%) had typical acute cellular rejection (tACR) using Banff criteria. Six (21\%) had hepatitic acute cellular rejection overlapping with typical features of acute cellular rejection; seven (25\%) had infiltrative acute cellular rejection (iACR) overlapping with typical features. The iACR occurred later than the tACR (124 versus 50 days; $P=0.032)$ and had a higher rise in baseline aspartate

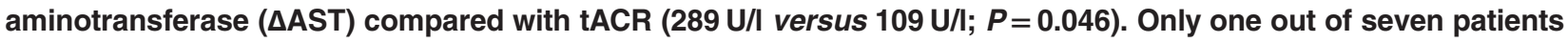
with iACR (14 versus $40 \%$ in tACR) failed Solu-Medrol boluses and required thymoglobulin. Patients with hepatitic acute cellular rejection (hACR) had similar $\triangle$ AST $(P=0.12)$ but higher bilirubinemia than typical acute cellular rejection (tACR) $(160 \mu \mathrm{mol} / \mathrm{l}$ versus $35 \mathrm{~mol} / \mathrm{l} ; \boldsymbol{P}=\mathbf{0 . 0 3 9})$ and required thymoglobulin in four out of six $(67 \%$ versus $40 \%$ ) instances. Patients with iACR had higher $\triangle A S T$ than tACR but better Solu-Medrol response compared with both tACR and hACR. hACR is different from plasma cell-rich late-occurring cellular rejection in its pattern but similar in its poor Solu-Medrol response.

Modern Pathology (2015) 28, 1275-1281; doi:10.1038/modpathol.2015.84; published online 31 July 2015
\end{abstract}

Histopathological assessment of liver allograft biopsy has a pivotal role in the diagnosis and management of posttransplant recipients, including in the diagnosis and grading of acute cellular rejection. ${ }^{1}$ Reports of histopathological correlates of acute cellular rejection from the early days of liver transplantation had shown that vascular endothelium, bile duct epithelium, and hepatocytes could be affected. ${ }^{2}$ The Banff group subsequently published a grading system that allocates a numerical weight to define the severity of injury mainly to the vascular

Correspondence: Dr OA Adeyi, MD, FRCPC, Laboratory Medicine Program, University Health Network (TGH Site), Toronto General Hospital, 200 Elizabeth Street, Room 11E-206, Toronto, ON M5G 2C4, Canada.

E-mail: oyedele.adeyi@uhn.ca

Received 4 February 2015; revised 5 June 2015; accepted 6 June 2015; published online 31 July 2015 endothelium (portal and hepatic veins) and bile duct epithelium. ${ }^{3}$ Each parameter, based on the extent of involvement, is given a score between 0 and 3 , to arrive at a maximum possible rejection activity index of $9 .^{3}$ The Banff scale, however, has not been independently validated and its individual components have not been shown to predict graft survival or response to steroids. ${ }^{4}$ Nevertheless, the morphologic criteria described for acute cellular rejection by this system are widely accepted parameters even when its numerical weightings are not applied. Pathologists have recognized additional patterns of injury in acute cellular rejection after liver transplantation, not incorporated in the Banff criteria. ${ }^{1,5-7}$ One such pattern is plasma cell hepatitis variant, variably referred to as de novo autoimmune hepatitis, hepatitic/atypical late-onset acute cellular rejection, or isolated central perivenulitis. ${ }^{6,8,9}$ This variant in our and others' experience typically occurs 
beyond the first year of transplant, and is characterized by a hepatitic, plasma cell-rich, perivenularaccentuated histologic picture and relative steroid resistance, when seen in adult population..$^{5,8,9}$

Two other features of acute cellular rejection have been recognized but are rarely reported or studied to determine their clinical relevance. These patterns either demonstrate active sinusoidal infiltration or hepatitic component, different from plasma cell hepatitis both in histopathological and clinical features. To our knowledge, both these patterns occur in the first year post transplant. These acute cellular rejection patterns are characterized by the presence of activated lymphocytes infiltrating the hepatic sinusoids or parenchymal necroinflammation without a noticeable preference for the perivenular zone. This study reports the clinico-pathological characteristics of our patient cohorts exhibiting these additional alloimmune injury patterns of acute cellular rejection, defined, respectively, as: sinusoidal iACR and hACR, which, although not included in the current Banff or any other grading system, appear to have significant clinical implication, including response to standard therapeutic interventions.

\section{Materials and methods}

Patients receiving liver transplants from 2007 to 2012 at the University Health Network, Toronto, Ontario, and who had biopsy-proven acute cellular rejection in the first year post transplant, were identified and corresponding liver biopsies reviewed. Liver allograft biopsies with acute cellular rejection of rejection activity index 5 or higher based on Banff grading were included. Milder forms of acute cellular rejection (rejection activity index 3 and 4) were excluded because the features of interest (ie iACR and hACR) have only been observed with moderate-to-severe acute cellular rejection. We also excluded patients transplanted for viral hepatitis B and $\mathrm{C}$, to eliminate the possibility of recurrent disease as a potential confounding factor. Patients with known or potential adverse drug reaction were also excluded. The remaining biopsies were reviewed independently by a senior gastrointestinal pathology fellow and an expert liver pathologist as follows: histopathological scoring was performed according to the Banff scale to assess tACR. In addition to the parameters included in the Banff criteria such as portal inflammation, bile duct injury, and (portal and/or hepatic) phlebitis, we documented the presence or absence of sinusoidal lymphocytic infiltrate, prominent plasma cells, hepatitis characterized by active necroinflammation, and cholestasis. Sinusoidal lymphocytic infiltrate is defined as liver biopsy in which sinusoids are infiltrated by activated lymphocytes (with lymphoblast or immunoblast features by H\&E and immunohistochemical staining for CD3 on representative cases) (Figure 1). Hepatitic features are defined as liver biopsy in which lobular and interface necroinflammation is seen (Figure 2). Significant plasma cell population was initially defined as plasma cells representing at $30 \%$ or more of the infiltrating cells. ${ }^{8}$ Having confirmed none of the included patients qualified for this, we further looked for more subtle $(10-30 \%)$ evidence of plasma cell prominence, but none met this less stringent criterion. Overall, we observed three distinct morphologic patterns of injury: The first group contained only tACR; the second group had tACR as well as significant sinusoidal lymphocytic infiltrates (iACR) as defined above (Figure 1); and the third group had tACR features plus a hepatitic component based on active parenchymal necroinflammation and interface activity (hACR) (Figure 2).

All patients in our study were on a tacrolimusbased regimen. There has been documentation of some patients having low tacrolimus troughs leading to acute cellular rejection episodes, but we have not further analyzed this in our cohort because the treatment data were incomplete in most of the patients. We, however, have no reasons to believe that these patients had risk factors for developing acute cellular rejection other than the risks in general transplant patients.

Clinical data were collected including time of acute cellular rejection relative to transplant date, treatment type and response, follow-up graft status at the time of study, and nature of original disease. Biochemical parameters such as change from baseline aspartate aminotransferase ( $\triangle \mathrm{AST})$, alkaline phosphatase $(\triangle \mathrm{ALP})$, and bilirubin at the time of acute cellular rejection and follow-up were analyzed.

\section{Statistical Analysis}

The data were expressed as means \pm standard error of mean. Differences were determined using Student's $T$-Test, with $P$ values $<0.05$ considered statistically significant.

\section{Results}

Twenty-eight biopsies from 28 patients were included (13 males and 15 females; Table 1). Of these, $15(54 \%)$ had tACR as described by the Banff criteria. Seven (25\%) had typical features plus prominent sinusoidal infiltration (iACR) by activated CD3-positive T cells (Figure 1); while six (21\%) had significant hepatitic inflammation (hACR) in addition to typical features (Figure 2). All patients had received similar tacrolimus- and prednisonebased immunosuppression with or without mycophenolate. Indications for transplantation include primary sclerosing cholangitis (9), primary biliary cirrhosis (4), alcohol cirrhosis (3), non-alcoholic steatohepatitis (3), autoimmune hepatitis cirrhosis (3), idiopathic cirrhosis (2), hepatocellular 

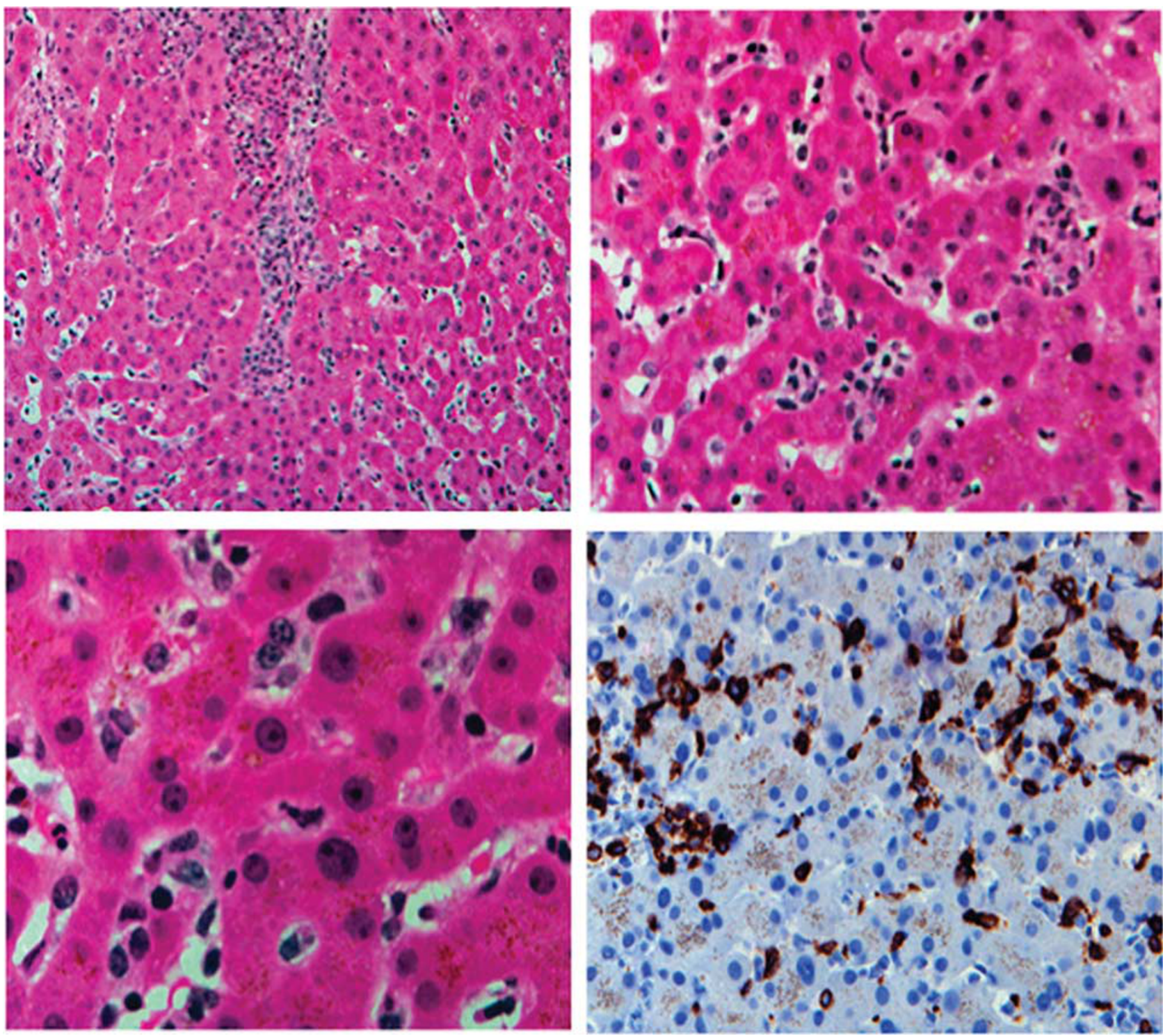

Figure 1 Prominent sinusoidal infiltration by activated CD3-positive cells in a patient with acute cellular rejection. This pattern is described as infiltrative pattern (hematoxylin and eosin (upper and left lower panels) and CD3 stains).

carcinoma, acute fulminant failure, Caroli's disease, and Budd-Chiari syndrome (Table 1). There is no apparent association between transplant indication on one hand and patterns or timing of acute cellular rejection on the other. The iACR occurred later than the tACR, with a mean of 124 days (range $=14-$ 254 days) versus 50 days (range $=6-228$ days) for tACR $(P=0.032)$. The hACR occurred from 6 to 190 days (mean $=64$ days), not significantly different from the tACR.

\section{Biochemical Parameters and Treatment Response}

Patients with iACR had higher $\triangle$ AST compared with the patients with iACR, with a mean $\triangle$ AST of $289 \mathrm{U} / \mathrm{l}$ for iACR rejection versus $109 \mathrm{U} / \mathrm{l}$ for tACR $(P=0.046)$ (Figure 3). Nevertheless, there was good response to standard Solu-Medrol boluses in all but one (6/7) iACR patients; and only one of the seven patients required thymoglobulin rescue (14 versus $40 \%$ in tACR). There was no difference in the baseline alkaline phosphatase between the iACR and the tACR $(P=0.4172)$.

Regarding hACR patients, the $\triangle \mathrm{AST}$ was not significantly different from tACR (hACR mean $=118$ $\mathrm{U} / \mathrm{l}$ versus tACR mean $=109 \mathrm{U} / \mathrm{l} ; P=0.12$ ) but presented with higher bilirubinemia than patients with tACR (mean 160 versus $35 \mathrm{mmol} / \mathrm{l} ; \quad P=0.039$ ) (Figure 3). Furthermore, these patients failed SoluMedrol boluses, requiring thymoglobulin rescue in four out of six (67\%) instances versus $40 \%$ of tACR patients who required thymoglobulin rescue. The number of patients in the hACR group was, however, too small to determine statistical significance of this parameter. There was no difference in the baseline 

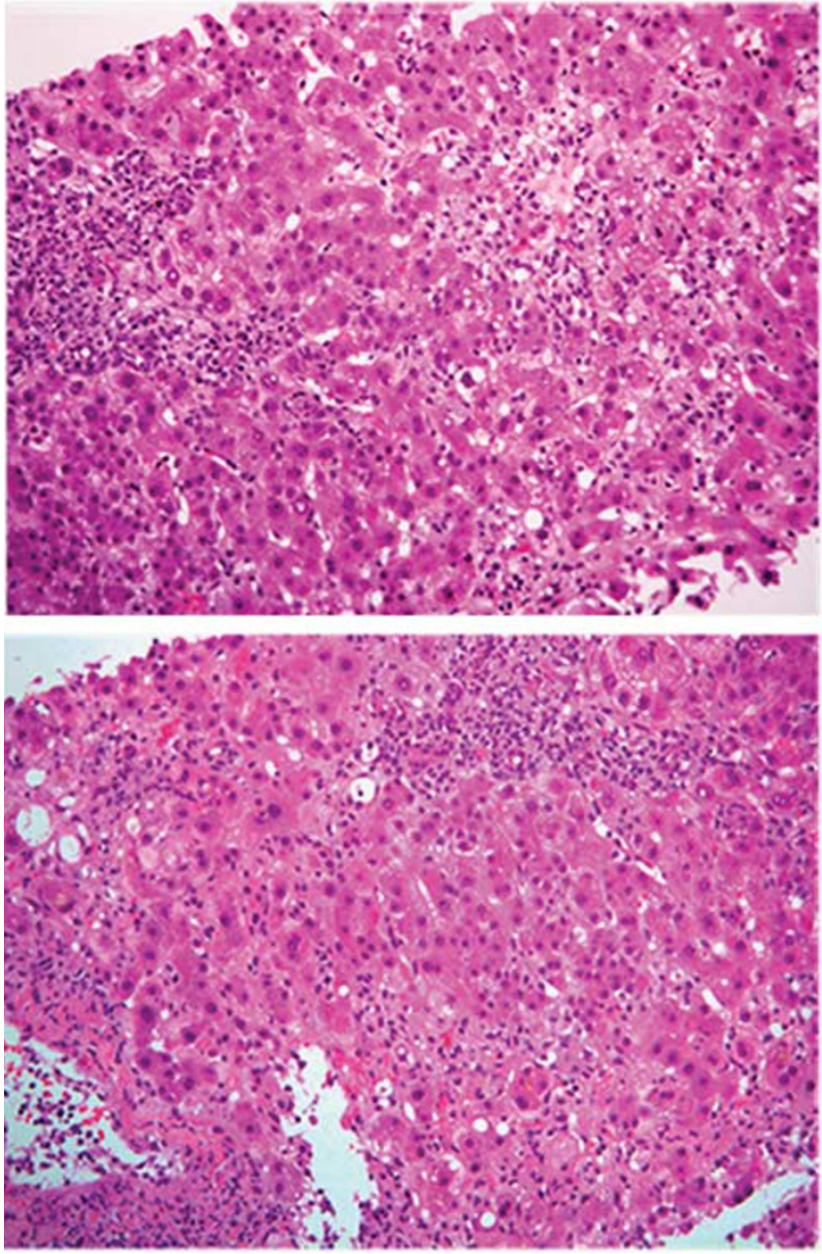

Figure 2 Hepatitic pattern overlapping with other typical features of acute cellular rejection. Note foci of confluent necroinflammation (hematoxylin and eosin stain (upper and lower panels)).

alkaline phosphatase between the hACR and tACR $(P=0.1644)$.

\section{Post-Acute Cellular Rejection Follow-up}

During the follow-up period, none of the patients with iACR developed chronic ductopenic rejection after the mean follow-up period of 1287 days (range $=207-2570$ days). One patient each in the hACR (mean follow-up of 925 days; range $=241-$ 1548 days) and tACR (mean follow-up of 969 days; range $=267-2023$ days) groups developed chronic ductopenic rejection.

\section{Discussion}

Acute cellular rejection following liver transplantation is a major cause of graft dysfunction, most occurring in the first 3-6 months post transplant, although it could also occur in later months and years, especially in patients with poor compliance and those in whom drug-drug interaction or other factors interfere with serum levels of calcineurin inhibitors. The typical histopathological features contributing to final acute cellular rejection severity grade have been well described, ${ }^{3,5}$ which dictate appropriate therapeutic interventions. ${ }^{1}$ In summary, these features include portal-based mixed inflammation consisting of activated lymphocytes, eosinophils, and a few plasma cells. Other features include evidence of bile duct epithelial and vascular (portal and hepatic venous and less frequently hepatic arterial) endothelial injuries. The sinusoidal endothelium is typically not regarded as an important target of alloimmune injury, however, there have been indirect evidence that it could be significantly injured as part of the acute cellular rejection process. ${ }^{10}$ Hepatocytes are believed to be 'privileged' in terms of alloimmune injury as reflected in most grading systems, which typically do not assess this compartment, except in cases of plasma cell hepatitis/de novo autoimmune hepatitis, when hepatocellular injury is believed to represent an 'atypical' form of rejection, or in patients with viral hepatitis C treated with interferon-based regimens. ${ }^{5,11,12}$

In the patient cohorts described above, we identified patients known to have acute cellular rejection with characteristic histological features of the same, and carefully removed all who could potentially have overlapping non-alloimmune injury, such as viral hepatitis and adverse reaction to medications, etc. We also limited our review to a time period where the hepatitic variant is typically rare, ie, in the first year post transplant. Left with a 'pure' group of acute cellular rejection patients, we documented the presence of active sinusoidal infiltration by, what we believe are alloimmune effector cells having activated lymphocyte features, and similar to portalbased effector cells responsible for bile duct epithelial and portal vein endothelial injuries. We also identified patients with hepatocellular necroinflammation not just in the periportal/interface areas, knowing that portal inflammation in acute cellular rejection could occasionally involve the interface hepatocytes, but throughout hepatic lobules with no particular predilection for the perivenular zone (Figure 2). Having confirmed that all these changes not only represented alloimmune injury for reasons above but also in that they all had other characteristic features of acute cellular rejection, we reviewed the clinical scenario around each patient. Our study confirmed that these additional patterns of alloimmune injury, iACR and hACR characterized patients with some important clinical differences from those with only the typical features of moderate-to-severe acute cellular rejection (tACR). For example, the patients with iACR responded better to standard Solu-Medrol therapies, rarely needing additional rescue with thymoglobulin, although they tend to occur outside the typical 3-month duration defined as 'early'. The excellent response to Solu-Medrol almost in all cases is interesting in this particular 


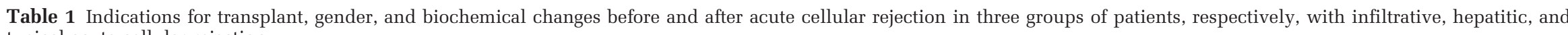
typical acute cellular rejection

\begin{tabular}{|c|c|c|c|c|c|}
\hline Indication for transplant & Gender & $\begin{array}{c}\text { Aspartate aminotransferase at time of } \\
\text { rejection }\end{array}$ & $\begin{array}{l}\text { Aspartate aminotransferase after } \\
\qquad R x\end{array}$ & $\begin{array}{l}\text { Bilirubin at time of } \\
\text { rejection }\end{array}$ & $\begin{array}{c}\text { Bilirubin after treatment for } \\
\text { rejection }\end{array}$ \\
\hline \multicolumn{6}{|c|}{ Patients with infiltrative pattern of acute cellular rejection a } \\
\hline Non-alcoholic steatohepatitis & Female & 334 & 69 & 95 & 12 \\
\hline Autoimmune hepatitis & Female & 178 & 265 & 12 & 125 \\
\hline Hepatocellular carcinoma without & Male & 736 & 26 & 43 & 9 \\
\hline \multicolumn{6}{|l|}{ cirrhosis } \\
\hline Alcohol & Female & 176 & 36 & 182 & 8 \\
\hline Primary biliary cirrhosis & Male & 320 & 29 & 21 & 13 \\
\hline Caroli's disease & Male & 34 & 13 & 9 & 7 \\
\hline Acute fulminant liver failure & Female & 92 & 31 & 24 & 11 \\
\hline \multicolumn{6}{|c|}{ Patients with hepatitic pattern of acute cellular rejection ${ }^{\mathrm{a}}$} \\
\hline Idiopathic cirrhosis & Male & 49 & 21 & 215 & 11 \\
\hline Alcohol & Female & 138 & 30 & 467 & 7 \\
\hline Acute Budd-Chiari & Female & 1851 & 25 & 119 & 9 \\
\hline Idiopathic cirrhosis & Male & 308 & 71 & 80 & 38 \\
\hline Primary sclerosing cholangitis & Female & 462 & 23 & 86 & 17 \\
\hline Primary sclerosing cholangitis & Male & 111 & 12 & 199 & 4 \\
\hline \multicolumn{6}{|c|}{ Patients with typical pattern of acute cellular rejection ${ }^{\mathrm{a}}$} \\
\hline Primary sclerosing cholangitis & Male & 94 & 27 & 59 & 14 \\
\hline Primary sclerosing cholangitis & Female & 99 & 15 & 182 & 10 \\
\hline Primary sclerosing cholangitis & Male & 485 & 16 & 33 & 7 \\
\hline Autoimmune hepatitis & Female & 71 & 21 & 19 & 7 \\
\hline Primary sclerosing cholangitis & Female & 267 & 30 & 85 & 8 \\
\hline Primary biliary cirrhosis & Female & 138 & 17 & 144 & 7 \\
\hline Non-alcoholic steatohepatitis & Male & 164 & 17 & 207 & 7 \\
\hline Primary sclerosing cholangitis & Female & 161 & 28 & 121 & 6 \\
\hline Primary sclerosing cholangitis & Female & 107 & 28 & 87 & 6 \\
\hline Autoimmune hepatitis & Female & 586 & 27 & 660 & 10 \\
\hline Non-alcoholic steatohepatitis & Male & 229 & 19 & 385 & 38 \\
\hline Primary biliary cirrhosis & Female & 70 & 16 & 303 & 6.5 \\
\hline Primary biliary cirrhosis & Male & 39 & 12 & 7 & 9 \\
\hline Primary sclerosing cholangitis & Male & 63 & 30 & 134 & 9.2 \\
\hline Alcohol & Male & 101 & 17 & 44 & 5 \\
\hline
\end{tabular}

${ }^{\mathrm{a} A s p a r t a t e}$ aminotransferase and bilirubin after treatment refer to values after Solu-Medrol or, in those requiring it, other rescue therapies. 

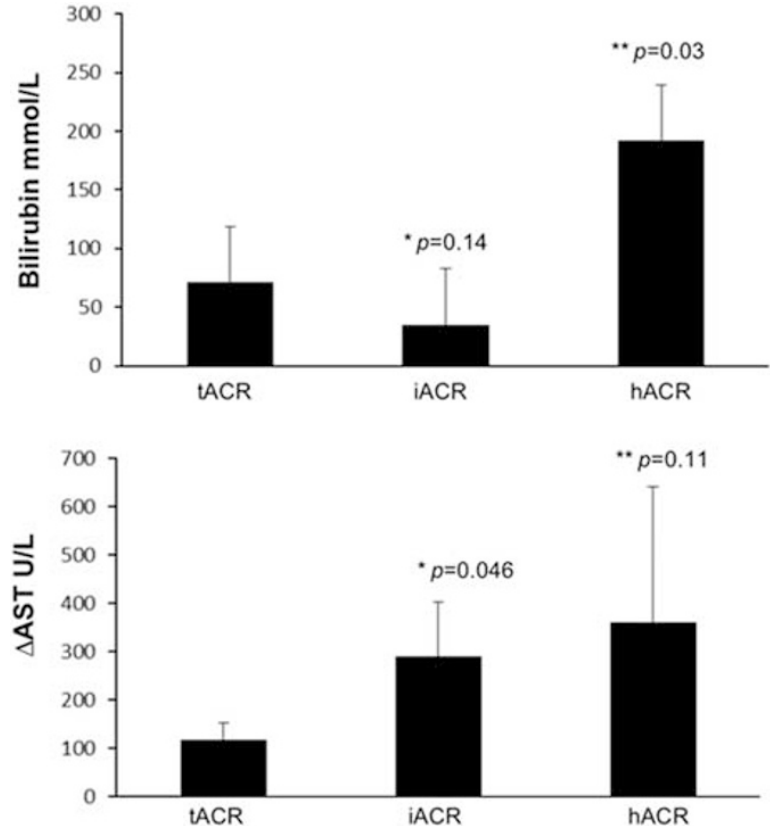

Figure 3 Charts comparing peak plasma levels of bilirubin and changes from baseline aspartate aminotransferase $(\triangle \mathrm{AST})$ in patients with typical (tACR) versus infiltrative (iACR) versus hepatitic (hACR) patterns of acute cellular rejection.

group, but we acknowledge that the small sample size constitutes a shortcoming and calls for further observation. On the other hand, most of the patients with hACR required thymoglobulin having failed to respond adequately or at all to standard Solu-Medrol boluses. As noted earlier, the iACR occurred between 3 and 6 months compared with the tACR and hACR, both of which occurred mostly before 2 months post transplant. Only two patients, one each from the tACR and hACR groups, developed chronic ductopenic rejection; but the patient numbers are rather too small and further studies will be necessary in order to determine the relevance of this observation.

The findings in this study highlight some important concepts, the first being how to record, grade, and communicate these additional findings to the treating hepatologist, because, among other weaknesses of the Banff system, it is not designed to assess these changes. Because, in some centers, the rejection activity index remains an important criteria for committing towards or away from aggressive intervention in liver allograft patients experiencing acute cellular rejection, we recommend adding a qualifier to pathology reports to indicate whether iACR and/or hACR features were present in addition to the features from which total rejection activity index was calculated: for example, Acute cellular rejection, RAI 6 /9 with hACR/iACR features. There is the potential that adding these features will improve the current poor Banff scoring performance for predicting steroid response or graft survival. ${ }^{4}$

The second concept, not addressed by our findings but clearly a relevant one is the role, if any, of alloantibodies in these infiltrative and hACR patterns. Although none of the patients in this cohort had been transplanted across ABO groups, and all were primary (first) transplants, unfortunately none had donor-specific antibody data available to enable us to address this question. However, we believe that there is at least some experimental evidence to suggest that hACR (and possibly iACR) should be a trigger for requesting donor-specific antibody information as part of clinical management in these patients. ${ }^{7}$ In their study, Sawada et $a l^{7}$ noticed the presence of C4d staining associated with hepatocellular/hepatitic injury in the biopsies of patients with moderate-to-severe acute cellular rejection, similar to our hACR cohort. However, the data of donorspecific antibody were not provided in the Sawada study. ${ }^{7}$ The collection of donor-specific antibody and other antibody-associated data when infiltrative and hACR patterns are present therefore has the potential to contribute to our still-evolving understanding of alloantibody-induced histopathological changes.

Lastly, there is the question of whether or not immunosuppression effectiveness prior to acute cellular rejection determined the pattern of acute cellular rejection. Although this study was not powered or designed to address this but all 27 patients had been on tacrolimus-based immunosuppression and while some had documented low serum tacrolimus levels in the preceding days/ weeks, there was no apparent difference in the frequency of these events among the three groups (assessed only in patients for whom this information was available). Nevertheless, additional studies would be needed to better understand the question of what role do the preceding periods of suboptimum immunosuppression have in determining the pattern of acute cellular rejection.

In conclusion, we have described two morphological patterns of acute cellular rejection that occur within the first year of liver transplantation and are associated with important clinical characteristics; including severity of injury as measured by increase in AST from baseline, response to treatment, and possibly, potential for chronicity; not assessed in the current Banff grading scale for the severity of acute cellular rejection. We recommend that when present, the iACR and hACR patterns should be reported in addition to the rejection activity index score in these patients primarily because of the therapeutic implications, but also to enable monitoring for progression to chronic rejection. These patterns could also be potentially utilized to trigger specific follow-up with donor-specific antibody, for which further studies are needed.

\section{Disclosure/conflict of interest}

The authors declare no conflict of interest. 


\section{References}

1 Hubscher SG. Transplantation pathology. Semin Liver Dis 2009;29:74-90.

2 Roddy H, Putnam CW, Fennell RH Jr. Pathology of liver transplantation. Transplantation 1976;22:625-630.

3 Banff schema for grading liver allograft rejection: an international consensus document. Hepatology 1997; 25:658-663.

4 Horoldt BS, Burattin M, Gunson BK et al. Does the Banff rejection activity index predict outcome in patients with early acute cellular rejection following liver transplantation? Liver Transpl 2006;12:1144-1151.

5 Adeyi O, Fischer SE, Guindi M. Liver allograft pathology: approach to interpretation of needle biopsies with clinicopathological correlation. J Clin Pathol 63:47-74.

6 Demetris AJ, Sebagh M. Plasma cell hepatitis in liver allografts: Variant of rejection or autoimmune hepatitis? Liver Transpl 2008;14:750-755.

7 Sawada T, Shimizu A, Kubota K et al. Lobular damage caused by cellular and humoral immunity in liver allograft rejection. Clin Transplant 2005;19:110-114.
8 Fiel MI, Agarwal K, Stanca C et al. Posttransplant plasma cell hepatitis (de novo autoimmune hepatitis) is a variant of rejection and may lead to a negative outcome in patients with hepatitis $\mathrm{C}$ virus. Liver Transpl 2008;14:861-871.

9 Krasinskas AM, Demetris AJ, Poterucha JJ et al. The prevalence and natural history of untreated isolated central perivenulitis in adult allograft livers. Liver Transpl 2008;14:625-632.

10 Usui M, Kuriyama N, Kisawada M et al. Tissue factor expression demonstrates severe sinusoidal endothelial cell damage during rejection after living-donor liver transplantation. J Hepatobiliary Pancreat Surg 2009;16: 513-520.

11 Guido M, Burra P. De novo autoimmune hepatitis after liver transplantation. Semin Liver Dis 2011;31: 71-81.

12 Merli M, Gentili F, Giusto M et al. Immune-mediated liver dysfunction after antiviral treatment in liver transplanted patients with hepatitis C: allo or autoimmune de novo hepatitis? Dig Liver Dis 2009;41: 345-349. 